BVL - Bundesvereinigung Logistik (Hrsg.): Wissenschaftssymposium Logistik der BVL 2002. München: Huss-Verlag GmbH, ISBN 3-9331724-61-1

\section{ISTAHC 2002: The Challenge of Cooperation}

\author{
Berlin, Germany, 9 - 12 June 2002
}

\section{von Matthias Perleth, AOK Bundesverband Berlin, Stabsbereich Medizin}

Wissenschaftliche Jahrestagungen sind Kristallisationspunkte des akademischen Lebens, die mehr oder weniger routiniert absolviert werden. Neueste Forschungsergebnisse und Konzepte werden präsentiert und diskutiert und es treffen sich Experten und alte Bekannte. Die jährlichen Tagungen der International Society of Technology Assessment in Health Care (ISTAHC) sind jedenfalls kein Routinebetrieb, dafür sorgen schon die jeweils wechselnden Organisatoren und der sich immer ändernde Tagungsort. Die ISTAHC versammelt die schnell wachsende Gemeinde der Experten für die medizinische Technologiebewertung (HTA).

Für die systematische Bewertung medizinischer Verfahren und Technologien in Bezug auf die Fragen, ob und unter welchen Umständen medizinische Technologien (darunter versteht man diagnostische, therapeutische, rehabilitative Verfahren, Organisationsstrukturen, Informations- und Supportsysteme) tatsächlich nützen, was ihre Einführung und breite Anwendung kosten und ob sie in den Leistungskatalog der gesetzlichen Krankenversicherung fallen sollen, hat sich auch im deutschsprachigen Raum der Begriff „Health Technology Assessment (HTA)" eingebürgert.

Die mittlerweile 18. Jahrestagung der ISTAHC fand vom 9. bis 12. Juni 2002 in Berlin statt, erstmals im deutschsprachigen Raum überhaupt. Die ISTAHC ist die einzige internationale wissenschaftliche Fachgesellschaft im Bereich HTA. Sie wurde 1985 gegründet, um Forschung, Ausbildung, Zusammenarbeit und den Informationsaustausch zu den klinischen, ökonomischen und gesellschaftlichen Implikationen von medizinischen Technologi- en zu fördern. Die Gesellschaft ist ein internationales Forum für alle, die mit der Evaluation von medizinischen Technologien befasst sind. Die ISTAHC hat gegenwärtig mehr als 1.400 Mitglieder weltweit.

Die Jahrestagung 2002 wurde von Vertretern der vier deutschsprachigen Länder, Deutschland, Luxemburg, Österreich und Schweiz organisiert, daher das naheliegende Motto „The Challenge of Collaboration“. Schon das Organisationsteam der Tagung nahm das Motto der Zusammenarbeit ernst, brachte es doch Vertreter von Ministerien, Universitäten, Krankenkassen, Ärzteorganisationen und weiterer Institute unter einen Hut. Das Programm erschöpfte sich aber nicht im Austausch deutschsprachiger Anekdoten. Der Schwerpunkt lag in der internationalen vertikalen und horizontalen Vernetzung von Initiativen im Bereich der Technologiebewertung, der evidenzbasierten Medizin, der Leitlinienentwicklung und der evidenzbasierten Politikberatung. Netzwerke nehmen eine immer größere Bedeutung im Austausch und in der Nutzung von Informationen ein. Neben einem Netzwerk deutschsprachiger HTA-Experten gibt es, um nur die wichtigsten zu nennen, noch das International Network of Agencies for Health Technology Assessment (INAHTA) und die European Collaboration in Health Technology Assessment (ECHTA). Wie die Zusammenarbeit auf internationaler Ebene verbessert werden kann, wurde daher in einer Reihe von Veranstaltungen diskutiert. Ein wichtiger Ansatz ergibt sich aus einer Verbesserung von strukturierten Zusammenfassungen von HTA-Berichten, um deren Austauschbarkeit zu gewährleisten. Diese $\mathrm{Zu}$ sammenfassungen sollten in Englisch abgefasst sein und so viele Informationen enthalten, dass die Ergebnisse übernommen werden können, ohne den kompletten Bericht in der Originalsprache lesen zu müssen. Eine wichtige Voraussetzung dafür ist jedoch eine nachvollziehbare und systematische Methodik.

In insgesamt 30 wissenschaftlichen Sitzungen und 20 Veranstaltungen mit eingeladenen Referenten diskutierten die rund 700 Teilnehmer aus 47 Ländern die neuesten Forschungsergebnisse und Konzepte zu HTA. Hochrangige Repräsentanten der Gesundheitsministerien in Deutschland, Luxemburg, Österreich und der Schweiz eröffneten die Tagung im Rahmen 
einer Plenarveranstaltung. Das deutsche Gesundheitsministerium wurde durch die parlamentarische Staatssekretärin Gudrun SchaichWalch vertreten. In bemerkenswerter Übereinstimmung betonten die Vertreter die Bedeutung von HTA für die Gestaltung der Gesundheitssysteme und stellten die teils weitgehenden gesetzlichen Regelungen vor oder kündigten solche an. Beklagt wurde auch die einseitige Informationspolitik der Industrie, der man ,die Stirn bieten müsse" (Otto Piller, Schweiz). Schaich-Walch stellte den vom Bundesgesundheitsministerium bereits 1995 angestoßenen Aufbau von HTA-Strukturen in Deutschland vor. Zunächst wurde die Methodik der Technologiebewertung im Rahmen eines universitären Forschungsprojekts entwickelt, seit 2001 ist das Deutsche Institut für Medizinische Dokumentation und Information (DIMDI) im Geschäftsbereich des Gesundheitsministeriums dafür zuständig, ein Informationssystem Health Technology Assessment aufzubauen, das sich aus eigens in Auftrag gegebenen HTA-Berichten speist. Wesentlich für die Funktion von HTA im Gesundheitswesen ist jedoch die Nutzung in Entscheidungen der gemeinsamen Selbstverwaltung. So werden in den Bundesausschüssen systematisch HTA-Berichte zur Entscheidungsunterstützung genutzt und entsprechende $\mathrm{Ab}$ teilungen für HTA sind im Medizinischen Dienst der Spitzenverbände der Krankenkassen, in der Kassenärztlichen Bundesvereinigung und der Bundesärztekammer etabliert. HTA hat damit in Deutschland im internationalen Vergleich einen hohen Stellenwert.

Im Fokus waren auch unkonventionelle Heilmethoden und wie diese mit dem schulmedizinischen Instrumentarium zur Bewertung klinischer Effekte adäquat erfasst werden können. Aktuelle Analysen zeigen, dass mindestens $10 \%$ aller Patienten in den westlichen Industrienationen mindestens einmal jährlich alternative Heilmethoden in Anspruch nehmen. Eine Studie aus England konnte zeigen, dass Akupunktur als am weitesten verbreitete alternative Heilmethode bei bis zu $25 \%$ der Behandelten Nebenwirkungen und eine zeitweise Verstärkung der Symptome aufweist.

Ein weiteres, bisher kaum in HTA-Kreisen beachtetes Gebiet ist die Zahnmedizin, dem als weiterer Fokus Aufmerksamkeit zuteil wurde. Einerseits werden derzeit in der zahnmedizini- schen Effektivitätsforschung Methoden etabliert, die in der klinischen Medizin schon vor Jahren langweilig waren; andererseits führen die besonderen Bedingungen der Zahnheilkunde zu methodischen Entwicklungen, von denen auch andere Bereiche profitieren. So werden spezielle Studiendesigns notwendig, um beispielsweise verschiedene Behandlungsmethoden am gleichen Gebiss lege artis, d.h. im Rahmen einer randomisierten kontrollierten Studie untersuchen zu können. Vor allem aber die Bedeutung präventiver, zahnerhaltender Maßnahmen wurde diskutiert und Ansätze zur Modellierung langfristiger Nutzen und Kosten wurden vorgestellt.

Bereits lange im Vorfeld der Tagung waren die Mittel- und Osteuropäischen Länder (MOE) als weiterer Schwerpunkt der HTAAktivitäten ins Blickfeld gerückt. Eine Serie von speziellen Veranstaltungen wurden zum Themenkomplex Umstrukturierung der Gesundheitssysteme in diesen Ländern und die Rolle von HTA in diesem Prozess organisiert. Das Interesse an diesem Programmaspekt war entsprechend hoch und so war die polnische Delegation mit fast 50 Personen eine der größten während der Tagung. Der Einfluss von HTA ist in den verschiedenen Ländern dabei höchst unterschiedlich entwickelt, obwohl alle MOE-Länder Versicherungssysteme einführen. In Polen beispielsweise scheint sich ein HTAUmfeld zu entwickeln, in dem private Institute vor allem der Industrie zuarbeiten. Ein nationales Institut für Qualitätssicherung inklusive HTA existiert zwar seit einigen Jahren, ein deutlicher Einfluss auf die Entscheidungsfindung im polnischen Gesundheitswesen ist aber nicht abzusehen. In Rumänien, das auch vom AOK-Bundesverband bei der Etablierung von evidenzbasierter Medizin unterstützt wird, setzen Versuche auf der Ebene der zentralen Gesundheitsverwaltung an, den Leistungskatalog mit Hilfe von HTA-Ergebnissen zu steuern.

Auf große Resonanz stieß eine Panelveranstaltung zu Disease Management Programmen, in dem internationale Erfahrungen ausgetauscht wurden. Dabei zeigte sich, dass viele Erfahrungen, die in den USA gemacht wurden, auch für die Einführung in Deutschland erwartet werden können. Insbesondere die Diskreditierung als „Kochbuchmedizin“ und mangelnde 
Akzeptanz stellen Gefährdungen für den Erfolg der DMPs dar.

Die Tagung wurde flankiert vom europäischen Symposium zu klinischen Praxisleitlinien, Präkonferenzworkshops zu verschiedenen Themenbereichen sowie einem Postkonferenzkurs zur Implementation von HTA in Mittelund Osteuropäischen Ländern.

Das komplette Programm ist auf der Homepage der Tagung, www.istahc2002.de, dokumentiert.

\section{Servicekasten}

- The Challenge of Collaboration. Proceedings of the $18^{\text {th }}$ Annual Meeting of the International Society of Technology Assessment in Health Care. München, Jena: Urban \& Fischer, 2002.

- Bundesgesundheitsblatt - Gesundheitsforschung - Gesundheitsschutz 2001, Band 44: Schwerpunktheft „Health Technology Assessment".

- TA-Datenbank-Nachrichten, 10. Jg., Heft 1, März 2001: Schwerpunktthema „Health Technology Assessment" (S. 4-58); siehe http://www.itas.fzk.de/deu/tadn/tadn011/ inhalt.htm

》)

\section{TA'02: Technikgestaltung im 21. Jahrhundert}

Wien, Österreich, 27. Mai 2002

\section{Bericht über die zweite österreichische TA- Konferenz von Walter Peissl, Institut für Technikfolgen-Abschätzung der Österreichi- schen Akademie der Wissenschaften, Wien}

Das Institut für Technikfolgen-Abschätzung (ITA) hat nach dem großen Erfolg der ersten österreichischen TA-Konferenz im Mai 2001 beschlossen, dem Bedarf nach einer Plattform für sozialwissenschaftliche Technikforschung in Österreich Rechnung zu tragen und auch im Jahre 2002 eine interdisziplinäre Tagung zu veranstalten. Ging es auf der ersten Konferenz noch vornehmlich darum, die heterogene österreichische Wissenschaftslandschaft im Bereich sozialwissenschaftlicher Technikforschung zu aktivieren und Austausch zu ermöglichen, wurde für TA'02 ein Generalthema gewählt: ,Technikgestaltung im 21. Jahrhundert - Im Spannungsfeld von Markt, Regulierung und Partizipation “. Vor dem Hintergrund globalisierter Märkte und der EU-weiten Diskussion um Governance und Partizipation wurden auf dieser Konferenz Fragen der Technikgestaltung und der Einflussmöglichkeiten verschiedener Akteure auf diese diskutiert. Anhand von Beispielen aus unterschiedlichen Technologiefeldern (Informations- und Kommunikationstechnologien, Biotechnologie) und aus bestimmten gesellschaftlichen Problembereichen (Nachhaltigkeitsdebatte) wurde das Spannungsverhältnis von Marktmechanismus, politischer Einflussnahme und partizipativen Ansätzen beleuchtet.

Aufgrund des regen Interesses ausländischer, vor allem deutscher KollegInnen an TA'01 wurde für TA'02 der Call auch für WissenschaftlerInnen aus dem Ausland geöffnet eine Maßnahme, die gut angekommen ist. Von den 27 angenommenen Präsentationen waren ein Drittel aus dem Ausland.

Als Keynote-Speaker konnte Arie Rip vom Centre for Studies of Science, Technology and Society der Universität Twente gewonnen werden. Er referierte zum Thema „TA as an element in reflexive co-evolution of technology and society: one more visible hand?" Im theoretischen Rahmen der Ko-Evolution von Technik und Gesellschaft wird die Technikentwicklung als eine Abfolge von Variation und Selektion verstanden. Rip argumentierte vor diesem Hintergrund für seine beiden zentralen Thesen:

- Die Institutionalisierung von TA sei ein Indikator für die sich verändernde Ko-Evolution von Technik und Gesellschaft. Das Interesse an TA sei eher ein Effekt dieses Wandels denn ein unabhängiger Input.

- TA könne ein Input sein, wenn es die KoEvolution reflexiver gestaltete und näher an den Prozess der Technik(mit)gestaltung herankäme. 